Ciccarelli $\mathrm{O}$ et al.

in press: Lancet Neurology

Accepted: 14 November 2018

CONFIDENTIAL - DO NOT DISTRIBUTE

\title{
SPINAL CORD INVOLVEMENT IN MULTIPLE SCLEROSIS AND NEUROMYELITIS OPTICA
} SPECTRUM DISORDERS

Olga Ciccarelli, Jeffrey A Cohen, Stephen C Reingold, and Brian G Weinshenker on behalf of the participants in the International Conference on Spinal Cord Involvement and Imaging in Multiple Sclerosis and Neuromyelitis Optica Spectrum Disorders *

Department of Neuroinflammation, UCL Institute of Neurology, and National Institute for Health Research (NIHR) University College London Hospitals (UCLH) Biomedical Research Center, London, UK (Prof O Ciccarelli, MBBS, PhD)

Neurologic Institute, Cleveland Clinic, Cleveland, OH, USA (Prof JA Cohen MD)

Scientific \& Clinical Review Associates LLC, Salisbury, CT, USA (SC Reingold, PhD)

Department of Neurology, Mayo Clinic, Rochester, MN, USA (Prof BG Weinshenker MD)

Correspondence to:

Prof Jeffrey A Cohen, Neurologic Institute, Cleveland Clinic, Cleveland, OH 44195 USA cohenj@ccf.org

* Conference attendees are listed in the Supplementary Appendix

Title character count:

Abstract word count:

Word count:

References

Tables:

Panels
89 (with spaces)

145 (150 maximum)

4477 (4500 maximum)

100 (100 maximum) 
Figures:

Supplementary appendix
4

Conference attendees, Supplementary table 


\section{Abstract}

Spinal cord pathology is an important cause of disability in multiple sclerosis (MS) and neuromyelitis optica spectrum disorders (NMOSD). MS and NMOSD are distinguished from other disorders that cause myelopathy by characteristic laboratory and radiologic findings, e.g. gadolinium enhancement pattern. However, limitations in the sensitivity and specificity of spinal cord imaging and poor correlation with disability measures have impeded understanding the relationship between spinal cord pathology and clinical manifestations of MS and NMOSD. Nevertheless, studies of the pathologic features of MS and NMOSD have shown that quantitatively different mechanisms lead to differences in clinical course and pattern of permanent disability accrual in the two disorders. Better understanding of these mechanisms is necessary to develop more informative clinical measures, electrophysiological methods, biomarkers, and imaging techniques to detect and monitor spinal cord involvement in diagnosis and management of MS and NMOSD, and as clinical trial outcome measures. 


\section{Introduction}

Spinal cord involvement is common in multiple sclerosis (MS) and neuromyelitis spectrum disorders (NMOSD) and an important contributor to disability. ${ }^{1,2}$ However, spinal cord pathology has been difficult to detect, characterize, and quantify because of limitations in the sensitivity and specificity of clinical outcome measures, e.g. Expanded Disability Status Scale (EDSS) and Multiple Sclerosis Functional Composite (MSFC), and spinal cord MRI. These limitations have impeded efforts to correlate spinal cord involvement with clinical manifestations and integrate measures of spinal cord disease as prognostic and outcome measures in clinical practice and research studies. ${ }^{3}$

Several developments have advanced our understanding of spinal cord involvement in MS and NMOSD. The 2017 revision of the McDonald criteria for MS diagnosis re-emphasized the relevance of clinical and MRI manifestations indicating spinal cord involvement to fulfill dissemination in time or space. ${ }^{4}$ New technology now allows more comprehensive assessment of gait impairment, a key consequence of spinal cord involvement, than provided by traditional clinical outcome measures. ${ }^{5,6}$ Diagnostic biomarkers, such as aquaporin-4 (AQP4) antibodies have allowed better delineation of the clinical and pathologic features of NMOSD and differences compared to $\mathrm{MS} .^{2}$ Neurofilament light chain concentration (NfL-c) in CSF and blood has emerged as useful biomarker of axonal damage, including in spinal cord, facilitating disease monitoring. ${ }^{7}$ Finally, new MRI techniques applied to the spinal cord show promise to elucidate clinical-pathological correlation and for use as clinical trial outcomes. ${ }^{3}$

This Review critically reviews clinical and radiologic manifestations of spinal cord involvement in MS and NMOSD; appraises clinical measures, electrophysiological methods, biomarkers, and imaging to detect and quantify spinal cord involvement; provides best practices for incorporating spinal cord imaging to assist with diagnosis and as a clinical trial outcome 
measure; considers aspects of MS and NMOSD pathophysiology that might explain differences in how disability accrues; and identifies key knowledge gaps and areas for future research.

\section{Clinical assessment of spinal cord involvement}

Clinical manifestations reflecting spinal cord involvement are common in MS and NMOSD. ${ }^{1}$ Motor impairment of the upper and lower extremities due to weakness, incoordination, or sensory loss, and gait impairment are characteristic of spinal cord involvement in MS and NMOSD. ${ }^{1}$ Both the EDSS and MSFC, the most commonly used MS-related disability measures, ${ }^{8}$ capture neurological manifestations that localize to the spinal cord. ${ }^{1}$ Neither has been validated in NMOSD. The Opticospinal Impairment Score is an ordinal scale quantifying optic nerve and spinal cord impairment in NMOSD. ${ }^{9}$ It is not widely used, and its psychometric properties have not been studied. The American Spinal Cord Injury Association (ASIA) impairment scale, a widely used 5-point ordinal scale to assess spinal cord injury, ${ }^{10}$ may be helpful to assess NMOSD patients. Quantitative measures potentially more sensitive to spinal cord damage than EDSS and MSFC include grip strength measured using a dynamometer and postural stability measures, ${ }^{11}$ but these are not commonly used in clinical practice or clinical trials. Neither of these measures has been fully validated, particularly for longitudinal assessment.

Gait impairment, a common sequel of spinal cord disease, is quantified in the EDSS by evaluating the distance a patient can walk and the assistance device required and in the MSFC by the Timed 25-Foot Walk, a test of short-distance walking speed. ${ }^{8}$ Measures of other aspects of walking and mobility include Timed Up and Go (ability to rise from sitting to standing, walk a short distance, and turn), 2- and 6-Minute Walk Tests (speed and endurance over an intermediate distance), and Multiple Sclerosis Walking Scale-12 (a patient self-report measure of walking ability). ${ }^{8}$ Formal gait analysis requires specialized technology but is more sensitive to 
mild changes in gait and response to interventions than standard neurologic testing. ${ }^{5}$ Subtle gait disturbance sometimes can be detected in MS before clinical signs are observed. ${ }^{5}$ Sensitivity may be increased by administration of a simultaneous cognitive task. ${ }^{12}$

Movement monitors that assess whole-body joint kinematics, spatial and temporal gait characteristics, and balance during walking and stairs climbing are replacing non-portable motion analysis systems. ${ }^{6}$ Studies employing wireless inertial gait analysis show that MS patients with minimal gait impairment can have abnormal postural sway ${ }^{13}$ or lower limb dysfunction. ${ }^{14}$ Advantages include small size and weight, and ability to collect, process, and rapidly analyze data from multiple sensors. The principal utility of these tools is more continuous monitoring of walking in a real-world environment to detect fluctuations during the day and trends over time. These measures capture different aspects of walking and mobility. Which aspect is most informative in MS and NMOSD at different levels of disability requires further study.

Neuropathic pain and bladder, bowel, and sexual dysfunction are common with spinal cord involvement in MS and NMOSD and decrease quality of life. ${ }^{15-18}$ Permanent bladder or erectile dysfunction out of proportion to motor or sensory sequelae is particularly suggestive of caudal spinal cord involvement characteristic of myelin oligodendrocyte glycoprotein (MOG) antibody-associated disease. ${ }^{19}$ The EDSS bladder/bowel functional system score and several questionnaires capture patient self-reported bladder, bowel, and sexual dysfunction. ${ }^{8}$ Specialized testing is required for quantitative assessment.

\section{Evoked potentials}

Somatosensory evoked potentials (SSEPs) and motor evoked potentials (MEPs) detect pathology involving the dorsal and lateral columns in the spinal cord, respectively, although slowed conduction and amplitude loss are nonspecific pathologically. ${ }^{20}$ Because multimodal 
$(\mathrm{mm})$ EPs, correlate with the EDSS both cross-sectionally and longitudinally ${ }^{21}$ and predict disease progression over three ${ }^{21}$ and 20 years, ${ }^{22}$ they merit consideration as outcome measures in phase 2 clinical trials to demonstrate reduced worsening with neuroprotective strategies or improvement with repair strategies. ${ }^{23}$ Use of mmEPs in MS multicenter trials studies requires standardization to reduce variability over time and between centers, and shortening acquisition time to reduce burden on subjects. Absence of mmEP responses in patients with advanced MS may preclude using lower extremity SSEPs and MEPs; assessment of the upper extremities may be informative in this situation. ${ }^{20}$

Asymptomatic EP abnormalities are uncommon in NMOSD, and reduced or absent responses are more common than slowed latency. ${ }^{24}$ The severity of abnormalities of lower extremity SSEPs and MEPs correlate with the severity of relapses in NMOSD. ${ }^{25}$ Further studies are needed to assess the ability of mmEPs to distinguish optic neuritis or myelitis in NMOSD versus MS, and their usefulness for monitoring NMOSD over time in clinical practice and clinical trials.

\section{Biomarkers}

A sensitive and specific biomarker, AQP4 antibodies, has greatly facilitated diagnosing NMOSD and defining previously unrecognized clinical features. ${ }^{2}$ The diagnosis and characterization of anti-MOG antibody disease also is rapidly evolving. ${ }^{26}$ There is no comparable diagnostic biomarker for MS, a major unmet need. The presence of CSF-specific oligoclonal bands supports the diagnosis of MS and argues against other disorders, including NMOSD. ${ }^{27}$ The $^{2}$ 2017 revision of the McDonald diagnostic criteria allows CSF-specific oligoclonal bands to substitute for dissemination in time in patients with a clinically isolated syndrome (CIS) and dissemination in space shown clinically or by MRI. ${ }^{4}$ 
The best validated non-imaging biomarker to monitor disease activity in MS and NMOSD is NfL-c in CSF and blood. ${ }^{7} \mathrm{NfL}-\mathrm{c}$ reflects ongoing axonal damage irrespective of cause or anatomic location. In MS, NfL-c relates most closely to inflammatory lesion activity and, to a lesser extent, the neurodegeneration underlying progression. ${ }^{7}$ In a longitudinal observational study of 259 patients with MS, baseline blood NfL-c at was positively associated with spinal cord volume loss, relapse, and disability worsening over two and five years. ${ }^{28}$ Blood NfL-c also shows promise as a treatment response marker; patients on disease therapy have lower levels than untreated patients and levels are reduced when in patients switch from low efficacy to higher efficacy therapy. ${ }^{29,30} \mathrm{NfL}$ levels have been studied in NMOSD and anti-MOG antibody disease. CSF NfL-c is higher in NMOSD compared to MS and other neurologic diseases, ${ }^{31}$ reflecting the highly destructive pathophysiology. Blood NfL-c relates to the frequency and severity of NMOSD relapses. ${ }^{32}$

\section{Radiological assessment of spinal cord involvement}

\section{Diagnosis and differential diagnosis}

MS, NMOSD, and acute disseminated encephalomyelitis (ADEM) can cause acute myelitis, generally with worsening over days to weeks followed by stabilization or recovery. ${ }^{4}$ Continuing clinical deterioration beyond three weeks, particularly over months, suggests an alternate etiology, such as sarcoidosis, dural arteriovenous fistula, spinal cord tumor, or metabolic or paraneoplastic disorder. ${ }^{33}$ The differential diagnosis between these disorders is aided by differences in imaging findings (Table). Dummy references ${ }^{34-46}$

The spinal cord is one of four sites of radiologic involvement utilized in the McDonald MS diagnostic criteria to document dissemination in space in patients who present with a CIS suggestive of MS. ${ }^{4} \mathrm{New}$ or gadolinium-enhancing spinal cord lesions can be used to document dissemination in time. ${ }^{4}$ MS spinal cord lesions typically are wedge-shaped on axial images, 
ovoid on sagittal images, and usually less than one segment in length and rarely over three

(Figure 1). Lesions are most commonly located in the spinal cord periphery (mainly posteriorly and laterally) but can involve central gray matter. Spinal cord lesions are particularly helpful for differential diagnosis because they do not occur in most common neurological conditions, such as migraine and cerebrovascular disorders, which are also associated with multifocal T2hyperintensities in the cerebral white matter and sometimes misdiagnosed as $\mathrm{MS} .{ }^{47}$ Detection of multiple peripheral asymmetric lesions in the spinal cord is almost pathognomonic of MS. Occasionally, in patients with progressive MS, diffuse spinal cord involvement mimicking a longitudinally extensive spinal cord lesion leads to diagnostic uncertainty. ${ }^{48}$ However, highresolution axial MRI usually demonstrates that the apparently confluent lesion comprises multiple discrete lesions.

Different from MS, NMOSD and ADEM produce longitudinally extensive spinal cord lesions, which can be similar in appearance. ${ }^{49} \mathrm{MOG}$ antibody-associated disease also can present with longitudinally extensive spinal cord lesions, which occur in the caudal spinal cord more often than in AQP4 antibody-associated disease. ${ }^{19}$ NMOSD lesions typically involve the central spinal cord and initially may have a linear appearance later evolving to become longitudinally extensive. ${ }^{50}$ So-called "bright spotty objects" - persistent areas of marked T2hyperintensity within lesions, which may have similar or higher intensity than CSF on T2weighted images, but not as low intensity as the CSF on T1-weighted images - are characteristic NMOSD spinal cord lesions. ${ }^{37}$ Acute NMOSD spinal cord lesions are hypointense on T1 images, which is rare in MS when using a T1 spin echo sequence. Chronic lesions in NMOSD, MS, and other conditions, including spinal cord infarction, may be T1 hypointense. ${ }^{35}$ Diagnostic criteria have used extension over more than three contiguous vertebral segments to indicate NMOSD. ${ }^{2}$ However, approximately $15 \%$ of untreated NMOSD patients with acute myelitis have lesions shorter than three vertebral segments, which can lead to a misdiagnosis of MS (Figure 2). ${ }^{51}$ In such cases, the diagnosis should be based on clinical, 
demographic, and serologic features. Conversely, longitudinally extensive spinal cord lesions have been reported in up to $10 \%$ of pediatric MS patients, ${ }^{52}$ though it is possible, in retrospect, some children in this series had MOG antibody-associated disease. ${ }^{19}$ Chronic NMOSD lesions may be non-specific in appearance and shorter than three vertebral segments. ${ }^{53}$

Acute NMOSD lesions almost always demonstrate gadolinium-enhancement, most often patchy, irregular, and more prominent in the margin. Although ring enhancement may be seen in both MS and NMOSD spinal cord lesions, ${ }^{34}$ it distinguishes NMOSD from other etiologies of longitudinally extensive myelopathy (Table, Figure 3). ${ }^{33}$ In particular, ring-enhancement is rarely encountered in sarcoidosis, spondylotic myelopathy, dural arteriovenous fistula, spinal cord infarction, and paraneoplastic myelopathy. ${ }^{34}$ Linear dorsal subpial enhancement over two vertebral segments is characteristic of sarcoidosis, though it also can occur in vitamin B12 deficiency. ${ }^{54}$

In summary, no single radiologic finding or combination is pathognomonic of a specific etiology. Synthesis of clinical and radiologic factors, and testing for AQP4 and MOG autoantibodies can yield a specific diagnosis in many patients who would have been classified as "idiopathic transverse myelitis" previously. ${ }^{55}$

\section{MRI to detect asymptomatic spinal cord lesions}

Brain MRI is useful to detect subclinical disease activity in $M S ;{ }^{.6}$ the role of spinal MRI to monitor for asymptomatic spinal cord lesions in MS and NMOSD is less clear. Asymptomatic spinal cord lesions are common in CNS demyelinating disease, including $35 \%$ of patients with radiologically isolated syndrome (RIS),${ }^{57} 27-53 \%$ of patients with non-spinal $\mathrm{CIS},{ }^{58}$ and $83 \%$ of patients with early relapsing-remitting $(\mathrm{RR}) \mathrm{MS} .{ }^{59}$ In patients with RIS, asymptomatic spinal cord lesions are associated with increased risk of becoming symptomatic, particularly with a progressive course. In a longitudinal study of RIS patients, all 15 patients who developed progressive symptoms from onset (i.e. PPMS) had spinal cord lesions at first detection versus 
72 of $113(64 \%)$ patients whose first clinical manifestation was an attack. ${ }^{57}$ In patients with nonspinal CIS, the number of asymptomatic spinal cord lesions predicts the risk of a second clinical event, ${ }^{60}$ and disability at two- ${ }^{60}$ and five-year follow-up. ${ }^{61}$ Thus, spinal cord MRI is advisable in the evaluation of patients with CIS.

In a longitudinal study of 103 patients with RRMS with median follow-up of 17 months, asymptomatic spinal cord lesions were detected in substantially fewer patients compared to brain, $25 \%$ and $44 \%$, respectively. ${ }^{62}$ Only $10 \%$ of patients had asymptomatic lesions in the spinal cord but not brain. Thus, spinal cord MRI may be less useful than brain MRI for routine clinical monitoring of RRMS patients.

Asymptomatic spinal cord lesions occur in NMOSD but are less common than in MS, and their ability to predict future disease activity and disability is unclear. ${ }^{63}$ Additional longitudinal studies are needed in MS and NMOSD to clarify the incidence of new asymptomatic lesions in spinal cord compared to brain, their impact on disease course, and implications for disease therapy.

\section{Technical aspects of imaging relevant for research clinical practice}

Spinal cord MRI is not performed to monitor MS in clinical practice as frequently as brain MRI; it prolongs the imaging session and, as a result, cost. Because spinal cord MRI is particularly susceptible to several potential artifacts generated by susceptibility differences at tissues interface, cerebrospinal (CSF) pulsation, respiratory motion, and swallowing, it is technically more challenging to obtain good quality images. Methods to mitigate such artifacts have been developed. ${ }^{64}$

A protocol that images the whole spine is generally preferred. However, because MRI detects spinal cord lesions in MS most often in the cervical region, ${ }^{65}$ a single sagittal acquisition from cervical level 1 to the upper thoracic spinal cord might be adequate and would improve image resolution because of the smaller field of view compared with whole spinal cord 
coverage. The preferred protocol to detect focal lesions includes a combination of two sagittal sequences, either both T2-weighted sequences, such as fast spin echo (FSE) and short tau inversion recovery (STIR), which allows fat suppression, or T2-weighted and proton density (PD)-w. Alternatively, an FSE T2-weighted or STIR sequence can be combined with a T1weighted sequence designed to enhance the contrast between tissues with different T1 relaxation times, such as phase-sensitive inversion recovery (PSIR), which nulls the signal of normal white matter (Figure 1). The combination of T2-weighted with STIR or PSIR images may offer the greatest contrast between lesions and surrounding tissue and highest sensitivity and specificity for lesion detection in the cervical spinal cord. ${ }^{66}$

Lesions detected on sagittal images ideally should be confirmed by acquisition of axial images (Figure 1). Axial imaging covering the whole spinal cord can be acquired in an acceptable length of time with parallel imaging acceleration and detects more lesions than sagittal imaging, ${ }^{65}$ especially small lesions in the spinal cord periphery. ${ }^{67}$ In some cases, the acquisition of axial slices of the entire spinal cord over five minutes with 6-mm slice thickness allows rejection of equivocal abnormalities seen on sagittal images. ${ }^{65}$ If two sagittal sequences are not acquired, a biplanar protocol (T2-weighted sagittal and axial slices of the entire spinal cord) can be considered.

Follow-up scans do not routinely require gadolinium administration, especially if no lesions are detected. Gadolinium is retained in brain, although no harmful effects of CNS deposition have been identified to date. ${ }^{68}$ If lesions are seen, then gadolinium-enhanced (single dose, $0.1 \mathrm{mmol} / \mathrm{kg}$ body weight, minimum delay time: 5 mins) T1-weighted spin-echo sequences (either 2D or 3D) are advised, ${ }^{69}$ to assess dissemination in time for the diagnosis of $M S^{4}$ Conversely, an enhancement pattern atypical of MS may indicate an alternative diagnosis. 
Technical improvements in MRI acquisition and analysis have made advanced spinal cord imaging more feasible for research. At present, translation to a clinical setting will likely be limited to experienced research sites and selected clinical cases. Additional research is needed to incorporate the following techniques into multicenter clinical trials.

Myelin water imaging provides more pathological specificity for demyelination than standard MRI. While myelin content decreases in the normal-appearing white matter in MS, it is restricted to lesions in NMOSD. ${ }^{70}$ Magnetization transfer imaging is less pathologically specific than myelin water imaging, because it reflects demyelination, axonal loss, and cellular tissue changes, but is more sensitive. The acquisition sequence is readily available, and the data are relatively straightforward to analyze. Magnetization transfer ratio demonstrates MS-related abnormalities in the spinal cord that cannot be detected by standard MRI and better explains clinical heterogeneity and severity of disability. ${ }^{71}$

Diffusion-weighted imaging methods, such as diffusion tensor imaging (DTI), also provide quantitative parameters that are somewhat pathologically specific and correlate with clinical disability. ${ }^{72}$ DTI-derived parameters in lesions and normal-appearing spinal cord differ between MS and NMOSD, reflecting the more destructive disease process in NMOSD. ${ }^{73}$ Further work is needed to overcome technical challenges to acquiring standardized, high quality data across scanner manufacturers and sites. Advanced diffusion MRI acquisition and modeling methods, such as q-space imaging ${ }^{74}$ or neurite orientation dispersion and density imaging, ${ }^{75}$ could provide more accurate information on spinal cord microstructure than DTI.

MR spectroscopy measures tissue metabolite composition. $\mathrm{N}$-acetyl-aspartate level is reduced in acute MS lesions when compared with healthy controls, indicating neuronal dysfunction, which may partially recover over time, or loss. ${ }^{76}$ Lower myo-inositol/creatine values are detected within NMOSD lesions when compared with healthy controls and MS patients, ${ }^{77}$ suggesting astrocytic damage. An in vivo study at 3T with postmortem validation extended the 
metabolic profile of normal spinal cord to include neurotransmitters and antioxidants. ${ }^{78}$ Application of this new technique to spinal cord disease is needed.

Advanced imaging has been used to study the association between focal spinal cord damage in MS and sensory-motor impairment measures by column-specific clinical outcomes. Dorsal column CSF-normalized magnetization transfer signal correlates with vibration sensation, and the lateral column signal correlates with ankle strength. ${ }^{79}$ Diffusion MRI measures in the dorsal column correlate with vibration sensation, while diffusion measures in dorsal and lateral columns correlate with Timed 25-Foot Walk and 9-Hole Peg Test. ${ }^{80}$ A crosssectional study at 3T in 21 patients with early PPMS and 24 controls showed association between column-specific quantitative imaging parameters and postural stability and vibration sensation. ${ }^{74}$ These studies demonstrate that advanced spinal cord imaging detects clinically relevant in vivo changes potentially applicable to MS diagnosis and disease monitoring. These approaches may not be as applicable to NMOSD, where lesions are larger and less tractspecific.

\section{MRI-assessed spinal cord atrophy as a clinical trial endpoint}

Spinal cord atrophy quantification (measuring progressive reduction in the cross-sectional spinal cord area over time) is the most attractive advanced technique for translation to the clinic because of its association with disability in MS and NMOSD. ${ }^{3}$ In MS, spinal cord atrophy correlates with concurrent disability and predicts long-term outcome. ${ }^{81}$ The rate of atrophy is faster in the spinal cord than in the brain and in secondary progressive MS (2.2\% per year) versus patients with CIS without disease activity during follow-up or with early, mild RRMS (0.5\% per year).$^{61,81}$ While patients with RRMS demonstrate more brain atrophy compared to spinal cord atrophy, especially in gray matter, NMOSD patients have more spinal cord than brain atrophy, ${ }^{82}$ suggesting different pathogenic mechanisms. Spinal cord atrophy can occur in NMOSD without spinal cord lesions, ${ }^{83}$ suggesting the presence of subtle spinal cord pathology 
not visible on MRI or diffuse brain atrophy leading to spinal cord atrophy. The relation to spinal cord atrophy in NMOSD to disease activity and disability accrual is unknown.

Atrophy is unique among the spinal cord imaging measures in having been applied as a secondary outcome in progressive MS clinical trials (appendix). However, trials have failed to show a significant treatment benefit on this metric, which could be due to absence of efficacy of the intervention, enrollment of a non-informative study population, or the variability in the measurement techniques available at the time. These limitations could be reduced by modifying eligibility criteria and by conducting trials in a single or limited number of centers to permit more control on technical aspects of imaging. An informative sample size was enrolled in a singlecenter phase 2 trial in progressive MS by requiring recent onset of progressive disease, mild to moderate disability, documentation of progression in the previous one or two years, and modest spinal cord atrophy. ${ }^{84}$ While these more restrictive eligibility criteria may improve the sensitivity of spinal cord atrophy as an outcome measure, they introduce recruitment challenges.

Spinal cord atrophy can be assessed with a 3D T1-weighted sequence (with isotropic resolution $\leq 1 \mathrm{~mm}^{3}$ to reduce partial volume effect). Measurement of cross-sectional area of the upper cervical spinal cord is needed until automatic registration-based methods are available. Automated imaging techniques using registration of scans over time will increase the precision of spinal cord atrophy measurements, making detection of treatment effects more feasible. Acquisition of 3D T1 volumetric brain scans to quantitate both brain and upper spinal cord atrophy ${ }^{85}$ may obviate the need for additional spinal cord imaging and facilitate incorporation of spinal cord atrophy in clinical trials in the meantime.

In summary, spinal cord atrophy may be clinically relevant and sensitive for monitoring patients with progressive MS and could be considered as primary endpoint in phase 2 trials with careful consideration of the study population and methodological issues related to imaging. The role of spinal cord atrophy as an outcome in NMOSD clinical trials currently is unclear. Relapse frequency and severity remain the recommended endpoints for NMOSD clinical trials. ${ }^{86}$ 


\section{Pathophysiology of disability accrual}

Disability accrues in MS predominantly from progression (gradual worsening separate from relapses) ${ }^{87}$ In contrast, although severe and permanent spinal cord-related disability is common in NMOSD, gradual disability progression is rare. ${ }^{88}$ Pathological differences between MS and NMOSD could explain this difference in clinical course.

Factors that lead to progression in MS include acute inflammatory axonal injury and degeneration of chronically demyelinated axons in focal lesions and in white and gray matter that appears normal on standard $\mathrm{MRI}{ }^{89}$ Progressive disability worsening associated with a single demyelinating lesion of the spinal cord ("solitary sclerosis") illustrates that neurodegeneration in a single focal lesion may be sufficient to cause progressive motor impairment. ${ }^{90}$ The long axons in ascending and descending spinal cord pathways are particularly susceptible to axonal transection in acute inflammatory lesions and to degeneration in chronically demyelinated lesions (Figure 4).

The pathological mechanisms producing spinal cord damage in MS overlap those in brain but with several differences. Cortical demyelination in brain, usually not detectable with $\mathrm{MRI}$, is strongly associated with disability and may be related to meningeal inflammatory cell infiltrates. ${ }^{91}$ Iron accumulation has been associated with neurodegeneration and may lead to reactive oxygen radical generation. ${ }^{92}$ Mitochondrial dysfunction and impaired transport lead to reduced energy production and amplify the effects of reactive oxygen intermediates. ${ }^{93}$ Lesions are particularly common in perfusion watershed areas in the brain (i.e., white matter at the intersection of perfusion territories supplied by the anterior, middle, and posterior cerebral arteries), suggesting ischemia contributes to demyelination and neurodegeneration. ${ }^{94}$ As in brain, demyelination and neurodegeneration in the spinal cord are present in radiographically normal white and gray matter ${ }^{95}$ and may be associated with meningeal inflammation. ${ }^{96}$ 
However, the spinal cord appears relatively spared from effects of ischemia and iron accumulation. ${ }^{97}$

The apparent absence of gradual progression in NMOSD could be an observational artifact, if major disability from attacks obscures further worsening. More likely explanations are the severe acute axonal destruction in NMOSD lesions, scarcity of viable chronically demyelinated axons (Figure 4), and absence of cerebral cortical gray matter lesions. ${ }^{98}$ Evidence of subclinical brain or spinal cord injury in NMOSD is controversial. ${ }^{99}$ Diffuse white matter injury and cervical spinal cord atrophy have been described in NMOSD in patients without a history of myelitis. ${ }^{83}$ In contrast, retinal nerve fiber layer thinning detected by optical coherence tomography is rare in NMOSD without a history of optic neuritis. ${ }^{100}$ Thus, several quantitatively different mechanisms lead to differences in clinical course and pattern of permanent disability accrual in MS and NMOSD. Although the exact mechanisms are uncertain, degeneration of chronically demyelinated but initially viable axons, especially in the spinal cord, appears to be the major cause of gradually worsening disability in progressive MS. ${ }^{89}$ In contrast, degeneration acutely injured axons in NMOSD appears to be the principal mechanism of NMOSD-related disability, and progressive disability worsening is uncommon.

\section{Conclusions and future directions}

Spinal cord pathology underlies much of the disability in MS and NMOSD, but the mechanisms underlying disability accrual likely differ. ${ }^{87,88}$ While degeneration of chronically demyelinated axons may explain disability progression in $\mathrm{MS}^{89}$ the scarcity of cortical lesions and demyelinated but viable axons accounts for the absence of progression in NMOSD. ${ }^{98}$ The pathogenesis of spinal cord lesion formation and its relation to accrual of permanent disability in both disorders is among areas for further study (Panel) to help understand the origin and 
evolution of spinal-cord-related outcomes and better define the role of spinal cord assessment in diagnosis and disease monitoring.

More sensitive and better standardized methods to assess spinal cord-related clinical manifestations over time are needed to monitor disease course and response to therapy. Comprehensive quantitative assessment of gait and mobility is sensitive to subtle impairment, ${ }^{5}$ but currently is not routinely performed and needs further validation to be useful for longitudinal monitoring, both in clinical practice and multicenter therapeutic trials in MS and NMOSD. Electrophysiological approaches can detect and quantify subclinical spinal cord pathology ${ }^{20}$ but need better standardization and reproducibility for routine application to multicenter studies.

The time over which clinical manifestations evolve,$^{4}$ the associated imaging characteristics (especially gadolinium-enhancement pattern), ${ }^{49}$ and biomarkers (e.g. AQP4 and MOG autoantibodies) $)^{2,26}$ help distinguish myelopathy from MS, NMOSD, and other disorders. However, because clinical and radiologic features may overlap, future studies to identify novel imaging biomarkers to help differentiate disorders still are needed. Application of best practices for spinal cord MRI acquisition improves image quality. ${ }^{64}$ Spinal cord MRI helps predict prognosis in MS. ${ }^{60,61}$ Whether it is useful for assessing prognosis in NMOSD and for monitoring for subclinical activity in either disorder is less clear. ${ }^{62,63}$ Advanced spinal cord imaging techniques may improve pathologic specificity. ${ }^{70,79,80}$ However, implementation presents a number of technical challenges, limiting them currently to the research setting. Spinal cord atrophy is attractive as a measure of overall spinal cord damage in MS and NMOSD and already has been employed as an endpoint in several MS clinical trials, though with limited success. It may become more useful once optimized to the level of reproducibility of brain atrophy measurements. ${ }^{85}$

In sum, there is a need to improve and validate clinical, electrophysiological, biomarker, and imaging measures for spinal cord disease, especially across centers, to increase translation 
of spinal cord assessment into patient care and clinical trials. These knowledge gaps signal the need for close cooperation between investigators with a wide-ranging areas of expertise.

\section{Acknowledgements}

This review was motivated by the International Conference on Spinal Cord Involvement and Imaging in Multiple Sclerosis and Neuromyelitis Optica Spectrum Disorders held 18-20 May 2017 in Berlin Germany. The conference was convened under the auspices of the International Advisory Committee on Clinical Trials in Multiple Sclerosis. Both the Committee and the Conference were sponsored and supported by the US National Multiple Sclerosis Society and the European Committee for Treatment and Research in Multiple Sclerosis. All Conference participants (Appendix) were provided the opportunity to review a draft of the manuscript and suggest revisions prior to finalization. There was no involvement of the sponsors in the design of the Conference, or collection, analysis or interpretation of data involved in the publication nor in the writing of the manuscript nor the decision to submit it for publication. We thank, Alex Rovira (Unidad de Resonancia Magnética, Hospital Universitari Vall d'Hebron, Barcelona, Spain) for providing Figure 1, Eoin Flanagan (Department of Neurology, Mayo Clinic, Rochester, MN, USA) for providing Figure 3, and Claudia Lucchinetti (Department of Neurology, Mayo Clinic, Rochester, MN, USA) for providing Figure 4. This project was supported by researchers (OC) at the National Institute for Health Research University College London Hospitals Biomedical Research Centre.

\section{Contributors}

O Ciccarelli, JA Cohen, SC Reingold, and BG Weinshenker designed the program agenda for the international conference that served as the basis for this review, prepared the initial drafts of this manuscript, edited the manuscript, and approved the final version for submission. 


\section{Declaration of interests}

Dr. Ciccarelli reports grants from MS Society of Great Britain \& Northern Ireland, NIHR UCLH BRC, National MS Society, NIHR, the Spinal Cord Research Foundation, Rosetrees Trust, Progressive MS Alliance, Bioclinica \& GE Neuro, EU-H2020; has received consultancy fees from Novartis, Teva, Roche, Biogen, Merck, and personal fees and other from Neurology, nonfinancial support and other from Multiple Sclerosis Journal,

Dr. Cohen reports consultancy fees from Adamas, Celgene, Convelo, EMD Serono, Novartis, PendoPharm, speaking fees from Mylan and Synthon; and is Co-Editor of Multiple Sclerosis Journal - Experimental, Translational and Clinical.

Dr. Reingold reports personal fees from National Multiple Sclerosis Society, European Committee for Treatment and Research in Multiple Sclerosis, Ionis Pharmaceuticals, Opexa Therapeutics, Teva Pharmaceutical Industries, and TG Therapeutics ; personal fees and other from F. Hoffmann-LaRoche, Medday Pharmaceuticals SA, Medlmmune Inc, Merck Serono, Novartis; other from Observatoire Français pour la Sclérosis en Plaques, personal fees; and non-financial support from Scientific and Clinical Review Associates LLC.

Dr. Weinshenker reports personal fees from Novartis, Medlmmune, Alexion, Caladrius Biosciences, Biogen-Idec, Roivant, Brainstorm Therapeutics; Dr. Weinshenker has a patent NMO-lgG for diagnosis of neuromyelitis optica with royalties paid to RSR Ltd.; Oxford University; Hospices Civil de Lyon, MVZ Labor PD Dr. Volkmann und Kollegen GbR. 


\section{References}

1. Krieger SC, Lublin FD. Location, location, location. Mult Scler J 2018; 24(11): 1396-8.

2. Wingerchuk DM, Banwell B, Bennett JL, et al. International consensus diagnostic criteria for neuromyelitis optica spectrum disorders. Neurology 2015; 85(2): 177-89.

3. Kearney H, Miller DH, Ciccarelli O. Spinal cord MRI in multiple sclerosis - diagnostic, prognostic and clinical value. Nat Rev Neurol 2015; 11(6): 327-38.

4. Thompson AJ, Banwell BL, Barkhof F, et al. Diagnosis of multiple sclerosis: 2017 revisions of the McDonald Criteria. Lancet Neurol 2018; 17(2): 162-73.

5. Martin CL, Phillips BA, Kilpatrick TJ, et al. Gait and balance impairment in early multiple sclerosis in the absence of clinical disability. Mult Scler J 2006; 12(5): 620-8.

6. Bradshaw MJ, Farrow S, Motl RW, Chitnis T. Wearable biosensors to monitor disability in multiple sclerosis. Neurol Clin Pract 2017; 7(4): 354-62.

7. Khalil M, Teunissen CE, Otto M, et al. Neurofilaments as biomarkers in neurological disorders. Nat Rev Neurol 2018; 14(10): 577-89.

8. Cohen JA, Reingold SC, Polman CH, Wolinsky JS, for the International Advisory Committee on Clinical Trials in Multiple Sclerosis. Disability outcome measures in multiple sclerosis trials: current status and future prospects. Lancet Neurology 2012; 11(5): 467-76.

9. Wingerchuk DM, Hogancamp WF, O'Brien PC, Weinshenker BG. The clinical course of neuromyelitis optica (Devic's syndrome). Neurology 1999; 53: 1107-14.

10. Kirshblum S, Waring W. Updates for the International Standards for Neurological Classification of Spinal Cord Injury. Phys Med Rehabil Clin N Am 2014; 25(3): 505-17.

11. Melillo F, A. DS, Martire S, Malentacchi M, Matta M, Bertolotto A. Computerized posturography is more sensitive than clinical Romberg test in detecting postural control impairment in minimally impaired multiple sclerosis patients. Mult Scler Rel Dis 2017; 14: $51-5$. 
12. Kalron A, Dvir Z, Achiron A. Walking while talking - difficulties incurred during initial stage of multiple sclerosis disease process. Gait Posture 2010; 32(3): 332-5.

13. Solomon AJ, Jacobs JV, Lomond KV, Henry SM. Detection of postural sway abnormalities by wireless inertial sensors in minimlally disabled patients with multiple sclerosis: a casecontrol study. J Neuroeng Rehabil 2015; $12: 74$.

14. Motta C, Palermo E, Studer V, et al. Disability and fatigue can be objectively measured in multiple sclerosis. PLOS ONE 2016; 11(2): e0148997.

15. Knan N, Smith MT. Multiple sclerosis-induced neuropathic pain: pharmacological management and pathophysiological insights from rodent EAE models. Inflammopharmacol 2014; 22(1): 1-22.

16. De Carvalho FL, Gomes CM, Apostolos-Pereira SL, et al. Voiding dysfunction in patients with neuromyelitis optica spectrum disorders. Neurourol Urodyn 2016; 35(1): 39-43.

17. Methley AM, Mutch K, Moore P, Jacob A. Development of a patient-centred conceptual framework of helth-related quality of life in neuromyelitis optica: a qualitative study. Health Expect 2017; 20(1): 47-58.

18. Ysrraelit MC, Fiol MP, Gaitan MI, Correale J. Quality of life assessment in multiple sclerosis: different perception between patients and neurologists. Front Neurol 2017; 8: 729.

19. Sato DK, Callegaro D, Lana-Peixoto MA, et al. Distinction between MOG antibody-positive and AQP4 antibody-positive NMO spectrum disorders. Neurology 2014; 82: 474-81.

20. Leocani L, Rovaris M, Boneschi FM, et al. Multimodal evoked potentials to assess the evolution of multiple sclerosis: a longitudinal study. J Neurol Neurosurg Psychiatry 2006; 77(9): 1030-5.

21. Schlaeger R, D'Souza M, Schindler C, Grize L, Kappos L, Fuhr P. Electrophysiological markers and predictors of the disease course in primary progressive multiple sclerosis. Mult scler J 2014; 20(1): 51-6. 
22. Schlaeger R, Schindler C, Grize L, et al. Combined visual and motor evoked potentials predict multiple sclerosis disability after 20 years. Mult Scler J 2014; 20(10): 1348-54.

23. Hardmeier M, Leocani L, Fuhr P. A new role for evoked potentials in MS? Repurposing evoked potentials as biomarkers for clinical trials in MS. Mult Scler J 2017; 23(10): 130919.

24. Ohnari K, Okada K, Takahashi T, Mafune K, Adachi H. Evoked potentials are useful for diagnosis of neuromyelitis optica spectrum disorder. J Neurol Sci 2016; 364: 97-101.

25. Tsao WC, Lyu RK, Ro LS, et al. Clinical correlations of motor and somatosensory evoked potentials in neuromyelitis optica. PLoS ONE 2014; 9(11): e113631.

26. Cobo-Calvo A, Ruiz A, Maillart E, et al. Clinical spectrum and prognostic value of CNS MOG autoimmunity in adults: the MOGADOR study. Neurology 2018; 90(21): e1858-69.

27. Stangel M, Fredrikson S, Meinl E, Petzold A, Stuve O, Tumani H. The utility of cerebrospinal fluid analysis in patients with multiple sclerois. Nat Rev Neurol 2013; 9(5): 267-76.

28. Barro C, Benkert P, Disanto G, et al. Serum neurofilament as a predictor of disease worsening and brain and spinal cord atrophy in multiple sclerosis. Brain 2018; Epub ahead of print 30 May 2018 doi: 10.1093/brain/awy154.

29. Disanto G, Barro C, Benkert P, et al. Serum neurofilament light: a biomarker of neuronal damage in multiple sclerosis. Ann Neurol 2017; 81(6): 857-70.

30. Piehl F, Kockum I, Khademi M, et al. Plasma neurofilament light chain levels in patients with MS switching from injectable therapies to fingolimod. Mult Scler J 2018; 24(8): 104654.

31. Wang H, Wang C, Qiu W, Lu Z, Hu X, Wang K. Cerebrospinal fluid light and heavy neurofilaments in neuromyelitis optica. Neurochem Int 2013; 63(8): 805-8. 
32. Mariotto S, Farinazzo A, Monaco S, et al. Serum neurofilament light chain in NMOSD and related disorders: comparison according to aquaporin-4 and myelin oligodendrocyte glycoprotein antibodies status. Mult Scler J - ETC 2017; 3(4): 2055217317743098.

33. Kitley JL, Leite MI, George JS, Palace JA. The differential diagnosis of longitudinally extensive transverse myelitis. Mult Scler J 2012; 18(3): 271-85.

34. Zalewski NL, Morris PP, Weinshenker BG, et al. Ring-enhancing spinal cord lesions in neuromyelitis optica spectrum disorders. J Neurol Neurosurg Psychiatry 2017; 88(3): 21825.

35. Valsasina P, Aboulwafa M, Preziosa P, et al. Cervical cord T1-weighted hypointense lesions at MR imaging in multiple sclerosis: relationship to cord atrophy and disability. Radiology 2018; 288(1): 234-44.

36. Tanaka M, Tanaka K, Komori M, Saida T. Anti-aquaporin 4 antibody in Japanese multiple sclerosis: the presence of optic spinal multiple sclerosis without long spinal cord lesions and anti-aquaporin 4 antibody. J Neurol Neurosurg Psychiatry 2007; 78(9): 990-2.

37. Hyun JW, Kim SH, Jeong IH, Lee SH, Kim HJ. Bright spotty lesions on the spinal cord: an additional MRI indicator of neuromyelitis optic spectrum disorder? J Neurol Neurosurg Psychiatry 2015; 86(11): 1280-2.

38. Kim HJ, Friedemann P, Lana-Peixoto MA, et al. MRI characteristics of neuromyelitis optica spectrum disorder: an international update. Neurology 2015; 84(11): 1165-73.

39. Wang YG, Wang YQ, Qiu W, Hu XQ, Lu ZZ. Clinical characteristics of neuromyelitis optica spectrum disorders associated with syringomyelia. Zhonghua Yi Xue Za Zhi 2017; 97(29): 2302-5.

40. Kitley J, Waters $\mathrm{P}$, Woodhall M, et al. Neuromyelitis optica spectrum disorders with aquaporin-4 and myelin-oligodendrocyte glycoprotein antibodies. A comparative study. JAMA Neurol 2014; 71: 276-83. 
41. Zalewski NL, Rabinstein AA, Wijdicks EFM, et al. Spontaneous posterior spinal artery infarction: an under-recognized cause of acute myelopathy. Neurology 2018; 91(9): 414-7.

42. Goh C, Phal PM, Desmond PM. Neuroimaging in acute transverse myelitis. Neuroimaging Clin N Am 2011; 21(4): 951-73.

43. Flanagan EP, Kaufmann TJ, Krecke KN, et al. Discriminating long myelitis of neuromyelitis optica from sarcoidosis. Ann Neurol 2016; 79(3): 437-47.

44. Flanagan EP, Krecke KN, Marsh RW, Giannini C, Keegan BM, Weinshenker BG. Specific pattern of gadolinium enhancement in spondylotic myelopathy. Ann Neurol 2014; 67(1): 54-65.

45. Zalewski NL, Flanagan EP. Autoimmune and paraneoplastic myelopathies. Semin Neurol 2018; 38(3): 278-89.

46. Kister I, Johnson E, Raz E, Babb J, Loh J, Shepherd TM. Specific MRI findings help distinguish acute transverse myelitis of neuromyelitis optica from spinal cord infarction. Mult Scler Relat Disord 2016; 9: 62-7.

47. Geraldes $\mathrm{R}$, Ciccarelli $\mathrm{O}$, Barkhof $\mathrm{F}$, et al. The current role of $\mathrm{MRI}$ in differentiating multiple sclerosis from its mimics. Nature Reviews Neurology 2018; 14(4): 213.

48. Bergers E, Bot JC, van der Valk $\mathrm{P}$, et al. Diffuse signal abnormalities in the spinal cord in multiple sclerosis: direct postmortem in situ magnetic resonance imaging correlated with in vitro high-resolution magnetic resonance imaging and histopathology. Ann Neurol 2002; 51(5): 652-6.

49. Brownlee WJ, Hardy TA, Fazekas F, Miller DH. Multiple Sclerosis 1. Diagnosis of multiple sclerosis: progress and challenges. Lancet 2017; 389(10076): 1336-46.

50. Cai W, Tan S-H, Zhang L, et al. Linear lesions may assist early diagnosis of neuromyelitis optica and longitudinally extensive transverse myelitis, two subtypes of NMOSD. J Neurol Sci 2016; 360: 88-93. 
51. Flanagan EP, Weinshenker BG, Krecke KN, et al. Short myelitis lesions in aquaporin-4positive neuromyelitis optica spectrum disorder. JAMA Neurol 2015; 72(1): 81-7.

52. Pidcock FS, Krishnan C, Crawford TO, Salorio CF, Trovato M, Kerr DA. Acute transverse myelitis in childhood: center-based analysis of 47 cases. Neurology 2007; 68(18): 147480.

53. Tackley G, O’Brien F, Rocha J, et al. Neuromyelitis optica relapses: race and rate, immunosuppression and impairment. Multiple Sclerosis and Related Diseases 2016; I 7: 21-5.

54. Paliwall VK, Malhotra HS, R.N. C, Agarwal A. "Anchor"-shaped bright posterior column in a patient with vitamin B12 deficiency myelopathy. Postgrad Med J 2009; 85(1002): 186.

55. Zalewski NL, Flanagan EP, Keegan BM. Evaluation of idiopathic transverse myelitis revealing specific myelopathy diagnoses. Neurology 2018; 90(2): e96-e102.

56. Wattjes MP, Rovira A, Miller D, et al. Evidence-based guidelines: MAGNIMS consensus guidelines on the use of MRI in multiple sclerosis - establishing disease prognosis and monitoring patients. Nat Rev Neurol 2015; 11(10): 597-606.

57. Kantarci $\mathrm{OH}$, Lebrun $\mathrm{C}$, Siva A, et al. Primary progressive multiple sclerosis evolving from radiologically isolated syndrome. Ann Neurol 2016; 79(2): 288-94.

58. Sombekke MH, Wattjes MP, Balk LJ, et al. Spinal cord lesions in patients with clinically isolated syndrome: a powerful tool in diagnosis and prognosis. Neurology 2013; 80(1): 6975.

59. Bot JC, Barkhof F, Polman $\mathrm{CH}$, et al. Spinal cord abnormalities in recently diagnosed MS patients: added value of spinal MRI examination. Neurology 2004; 62(2): 226-33.

60. Arrambide G, Tintore M, Auger C, et al. Lesion topographies in multiple sclerosis diagnosis: a reappraisal Neurology 2017; 89(23): 2351-6. 
61. Brownlee WJ, Altmann DR, Alves Da Mota P, et al. Association of asymptomatic spinal cord lesions and atrophy with disability 5 years after a clinically isolated syndrome. Mult Scler J 2017; 23(5): 665-74.

62. Zecca C, Disanto G, Sormani MP, et al. Relevance of asymptomatic spinal MRI lesions in patients with multiple sclerosis. Mult Scler J 2016; 22(6): 782-91.

63. Flanagan EP, Weinshenker BG, Krecke KN, Pittock SJ. Aymptomatic myelitis in neuromyelitis optica and autoimmune aquaporin-4 channelopathy. Neurol Clin Pract 2015; 5(2): $175-7$.

64. Stroman PW, Wheeler-Kingshott C, Bacon M, et al. The current state-of-the-art of spinal cord imaging: methods. Neuroimage 2014; 84: 1070-81.

65. Weier K, Mazraeh J, Naegelin Y, et al. Biplanar MRI for the assessment of the spinal cord in multiple sclerosis. Mult Scler J 2012; 18(11): 1560-9.

66. Alcaide-Leon P, Pauranik A, Alshafai L, et al. Comparison of sagittal FSE T2, STIR, and T1-weighted phase-sensitive inversion recovery in the detection of spinal cord lesions in MS at 3T. AJNR Am J Neuroradiology 2016; 37(5): 970-5.

67. Breckwoldt MO, Gradl J, Hahnel S, et al. Increasing the sensitivity of MRI for the detection of multiple sclerosis lesions by long axial coveage of the spinal cord: a prospective study in 119 patients. J Neurol 2017; 264(2): 341-9.

68. US Food and Drug Administration. Drug safety communication. 2017. https://www.fda.gov/Drugs/DrugSafety/ucm589213.htm (accessed 22 October 2018.

69. Traboulsee A, Simon JH, Stone L, et al. Revised recommendations of the Consortium of MS Centers task force for a standardized MRI protocol and clinical guidelines for the diagnosis and follow-up of multiple sclerosis. AJNR Am J Neuroradiology 2016; 37(3): 394-401. 
70. Combes AJE, Matthews L, Lee JS, et al. Cervical cord myelin water imaging shows degenerative changes over one year in multiple sclerosis but not neuromyelitis optic spectrum disorder. Neuroimage Clin 2017; 16: 17-22.

71. Oh J, Saidha S, Chen M, et al. Spinal cord quantitative MRI discriminates between disability levels in multiple sclerosis. Neurology 2013; 80(6): 540-7.

72. Ciccarelli O, Wheeler-Kingshott C, McLean MA, et al. Spinal cord spectroscopy and diffusion-based tractography to assess acute disability in multiple sclerosis. Brain 2007; 130(Pt 8): 2220-31.

73. Klawiter EC, Xu J, Naismith RT, et al. Increased radial diffusivity in spinal cord lesions in neuromyelitis optica compared with multiple sclerosis. Mult Scler J 2012; 18(9): 1259-68.

74. Abdel-Aziz K, Schneider T, Solansky BS, et al. Evidence for early neurodegeneration in the cervical cord of patients with primary progressive multiple sclerosis. Brain 2015; 138(Pt 6): $1568-82$.

75. By S, Xu J, Box BA, Bagnato FR, Smith SA. Application and evaluation of NODDI in the cervical spinal cord of multiple sclerosis patients. Neuroimage Clin 2017; 15: 333-42.

76. Ciccarelli O, Altmann DR, McLean MA, et al. Spinal cord repair in MS: does mitochondrial metabolism play a role? Neurology 2010; 74(9): 721-7.

77. Ciccarelli O, Thomas DL, De Vita E, et al. Low myo-inositol indicating astrocytic damage in a case series of neuromyelitis optica. Ann Neurol 2013; 74(2): 301-5.

78. Hock A, Wilm B, Zandomeneghi G, et al. Neurochemical profile of the human cervical spinal cord determined by MRS. NMR Biomed 2016; 29(10): 1464-76.

79. Zackowski KM, Smith SA, Reich DS, et al. Sensorimotor dysfunction in multiple sclerosis and column-specific magnetization transfer-imaging abnormalities in the spinal cord. Brain 2009; 132(Pt 5): 1200-9.

80. Naismith RT, Xu J, Klawiter EC, et al. Spinal cord tract diffusion tensor imaging reveals disability substrate in demyelinating disease. Neurology 2013; 80(24): 2201-9. 
81. Tsagkas C, Magon S, Gaetano L, et al. Spinal cord volume loss. A marker of disease progression in multiple sclerosis. Neurology 2018; 91(4): e349-58.

82. Liu Y, Wang J, Daams M, et al. Differential patterns of spinal cord and brain atrophy in NMO and MS. Neurology 2015; 84(14): 1465-72.

83. Ventura RE, Kister I, Chung S, Babb JS, Shepherd TM. Cervical spinal cord atrophy in NMOSD without a history of myelitis of MRI-visible lesions. Neurol Neuroimmunol Neuroinflamm 2016; 3(3): e224.

84. Cawley N, Tur C, Prados F, et al. Spinal cord atrophy as a primary outcome measure in phase II trials of progressive multiple sclerosis. Mult Scler J 2018; 24(7): 932-41.

85. Liu Y, Lukas C, Steenwijk MD, et al. Multicenter validation of mean upper cervical cord area measurements from head 3D T1-weighed MR imaging in patients with multiple sclerosis. AJNR Am J Neuroradiology 2016; 37(4): 749-54.

86. Weinshenker BG, Barron G, Behne JM, et al. Challenges and opportunities in designing clinical trials for neuromyelitis optica. Neurology 2015; 84(17): 1805-15.

87. Ontaneda D, Thompson AJ, Fox RJ, Cohen JA. Progressive multiple sclerosis: prospects for disease therapy, repair, and restoration of function. Lancet 2017; 389(10076): 1357-66.

88. Wingerchuk DM, Pittock SJ, Lucchinetti CF, Lennon VA, Weinshenker BG. A secondary progressive clinical course is uncommon in neuromyelitis optica. Neurology $2007 ; 68(8)$ : 603-5.

89. Lassmann H, van Horssen J, Mahad D. Progressive multiple sclerosis: pathology and pathogenesis. Nat Rev Neurol 2012; 8(11): 647-56.

90. Keegan BM, Kaufmann TJ, Weinshenker BG, et al. Progressive solitary sclerosis. Gradual motor impairment from a single CNS demyelinating lesion. Neurology 2016; 87(16): 1713-9.

91. Calabrese M, Magliozzi R, Ciccarelli O, Geurts JJ, Reynolds R, Martin R. Exploring the origins of grey matter damage in multiple sclerosis. Nat Rev Neurosci 2015; 16(3): 147-58. 
92. Hametner S, Wimmer I, Haider L, Pfeifenbring S, Bruck W, Lassmann H. Iron and neurodegeneration in the multiple sclerosis brain. Ann Neurol 2013; 74(6): 848-61.

93. Mahad D, Ziabreva I, Campbell G, et al. Mitochondrial changes within axons in multiple sclerosis. Brain 2009; 132(Pt 5): 1161-74.

94. Haider L, Zrzavy T, Hametner S, et al. The topography of demyelination and neurodegeneration in the multiple sclerosis brain. Brain 2016; 139(Pt 3): 807-15.

95. Schirmer L, Antel JP, Bruck W, Stadelmann C. Axonal loss and neurofilament phosphorylation changes accompany lesion development and clinical progression in multiple sclerosis. Brain Pathol 2011; 21(4): 428-40.

96. Androdias G, Reynolds R, Chanal M, Ritleng C, Confavreux C, Nataf S. Meningeal T cells associate with diffuse axonal loss in multiple sclerosis spinal cords. Ann Neurol 2010; 68(4): $465-76$.

97. Haider L, Simeodidou C, Steinberger G, et al. Multiple sclerosis deep grey matter: the relation between demyelination, neurodegeneration and iron. J Neurol Neurosurg Psychiatry 2014; 85(12): 1386-95.

98. Sinnecker T, Dörr J, Pfueller CF, et al. Distinct lesion morphology at 7-T MRI differentiates neuromyelitis optica from multiple sclerosis. Neurology 2012; 79(7): 708-14.

99. Kawachi I, Lassmann H. Neurodegeneration in multiple sclerosis and neuromyelitis optica. J Neurol Neurosurg Psychiatry 2017; 88(2): 137-45.

100. Bennett JL, de Seze J, Lana-Peixoto M, et al. Neuromyelitis optica and multiple sclerosis: seeing differences through optical coherence tomography. Mult Scler J 2015; 21(6): 67888. 


\section{Panel: Search strategy and selection criteria}

In preparing this review, the authors conducted literature searches in PubMed (English language from January 1, 1980 through October 15, 2018 with a focus on papers since 2010 but including earlier publications as appropriate) using search terms: "multiple sclerosis", "neuromyelitis optica spectrum disorder", and "spinal cord disease", and the intersection of these disease descriptors with "clinical signs and symptoms", "assessment", "outcomes", “imaging", "electrophysiology", "biomarker", and "activity monitor". 


\section{Panel: Directions for future research}

- Further study of differences in the pathophysiology of MS and NMOSD to better elucidate the pathobiology of axonal loss and disability accrual

- Development and validation of better gait and mobility assessment tools, activity monitors and other paraclinical tools to detect and track spinal cord involvement

- Standardization and multicenter applicability of electrophysiology tools that assess spinal cord function longitudinally

- Incorporation of available clinical and imaging spinal cord assessments into routine patient monitoring and into outcomes used to evaluate novel therapies

- Incorporation of recently recognized imaging patterns and especially patterns of gadoliniumenhancement to facilitate differential diagnosis of inflammatory myelopathies and distinguish inflammation from spinal cord compression and infarction; assessment of these patterns in clinical practice

- Development of automated image registration techniques to increase precision and reduce variability of longitudinal (over time) spinal cord atrophy quantification 


\section{Figure Legends}

\section{Figure 1: Potential diagnostic protocols for spinal cord imaging in MS and NMOSD}

Three protocols suggested for use in the clinical setting are shown. Each protocol consists of two sagittal images, for example (A.a) T2 sagittal image and (A.b) STIR sagittal image, (B.a) T2 sagittal image and (B.b) PD sagittal image, or (C.a) T2 sagittal image and (C.b) PSIR sagittal image. If lesions are seen on sagittal scans, axial scans should be acquired. T2-weighted axial images are shown in (D).

\section{Figure 2: Short lesions in NMOSD}

A 39-year old male patient presented with imbalance and gait impairment. Spinal cord MRI obtained at the time of presentation showed a short lesion in the central spinal cord at the C5 level (left panel). Brain MRI showed lesions suggestive of inflammatory demyelination (not shown). Lumbar puncture demonstrated CSF-specific oligoclonal bands. In his history there were two possible episodes of optic neuritis and weakness in his legs occurring about 10 years earlier, when he was living abroad. He was diagnosed with relapsing-remitting MS and treated first with interferon-beta. Because of recurrent episodes of partial myelitis over the subsequent five years, treatment was changed to fingolimod. Over the following year, he experienced two further partial myelitis episodes. While off fingolimod, in preparation for escalating to monoclonal antibody therapy, he presented with weakness and numbness of all four limbs and urinary retention. Repeat spinal cord MRI during the episode of myelitis showed a longitudinally extensive lesion typical of NMOSD (center and right panels).

$\mathrm{CSF}=$ cerebrospinal fluid, $\mathrm{MRI}=$ magnetic resonance imaging, $\mathrm{MS}=$ multiple sclerosis NMOSD = neuromyelitis spectrum disorders. 


\section{Figure 3: Gadolinium enhancement patterns in varying myelopathy etiologies}

MS: short T2-hyperintense lesion on sagittal MRI extending one vertebral segment (A1) has accompanying ring enhancement on T1-post-gadolinium sagittal (A2, arrow) and axial (A3, arrow) sequences.

AQP4 antibody-associated NMOSD: a longitudinally extensive T2-hyperintense lesion on sagittal images (B1) demonstrates ring-enhancement on T1-post-gadolinium sagittal (B2, arrow) and axial (B3, arrow) sequences.

Sarcoidosis: a longitudinally extensive T2-hyperintense lesion on sagittal images (C1) has accompanying enhancement on T1-post-gadolinium sequences that extends in a linear fashion along the dorsal subpial surface (C2, C3; arrow) with occasional central canal enhancement also evident (C2, C3: arrowhead) that on axial sequences forms a trident-like pattern (C3). Cervical spondylosis: a longitudinally extensive T2-hyperintense lesion on sagittal sequence (D1) has accompanying enhancement on T1-post-gadolinium sequences that forms a transverse band (pancake-like) just below the site of maximal stenosis (B2, arrow) and involves the spinal cord white matter sparing gray matter on axial sequences (D3, arrows).

AQP4 = aquaporin-4, MRI = magnetic resonance imaging, $M S$ = multiple sclerosis

\section{Figure 4: Myelin and axonal pathology in NMOSD and MS}

NMOSD: Myelin PLP immunohistochemical staining of the spinal cord in NMOSD demonstrates demyelinating lesions (asterisk, (A)) with AQP4 loss on immunohistochemical staining (asterisk, (B)) and axonal loss by Bielschowsky silver staining (asterisk, (C)). An early pre-demyelinating lesion is characterized by myelin preservation (boxed area in (A) and (D)), AQP4 loss (boxed area in $(B)$ and $(E)$ ), and axonal preservation (boxed area in $(C)$ and $(F)$ ). 
MS: (G) and (I) PLP immunohistochemical staining shows a chronic demyelinating lesion in the spinal cord in MS. (H) and (I) Bielschowsky silver staining indicates axonal damage characterized with the decreased axons (the green dot line highlights border of the lesion).

Scale bars $=100 \mu \mathrm{m}$. AQP4 = aquaporin-4, MS = multiple sclerosis, NMOSD = neuromyelitis spectrum disorders, PLP $=$ proteolipid protein. 
Table: MRI characteristics of spinal cord lesions

\begin{tabular}{|c|c|c|c|c|c|}
\hline Diagnosis & $\begin{array}{l}\text { Lesion length and } \\
\text { location on sagittal } \\
\text { images }\end{array}$ & $\begin{array}{l}\text { Location on } \\
\text { axial images }\end{array}$ & $\begin{array}{l}\text { T2 signal } \\
\text { characteristics }\end{array}$ & $\begin{array}{l}\text { T1 signal } \\
\text { characteristics }\end{array}$ & $\begin{array}{l}\text { Gadolinium } \\
\text { enhancement }\end{array}$ \\
\hline Multiple sclerosis ${ }^{34,35}$ & $\begin{array}{l}\text { Usually <1 vertebral } \\
\text { segments; consistently } \\
<3 \text { vertebral segments }{ }^{a}\end{array}$ & $\begin{array}{l}\text { Multiple, } \\
\text { asymmetrical, }\end{array}$ & Hyperintense & $\begin{array}{l}\text { Isointense or } \\
\text { hypointense in } \\
\text { chronic lesions } \\
\text { studied with } 3 T \\
\text { MRI scanners, } \\
\text { especially in } \\
\text { patients with } \\
\text { progressive MS }\end{array}$ & $\begin{array}{l}\text { Present in most } \\
\text { acute lesions; } \\
\text { variable pattern: } \\
\text { homogeneous; } \\
\text { "ring-enhancing" } \\
\text { in } 20 \%\end{array}$ \\
\hline NMOSD (AQP4) & $\begin{array}{l}85 \% \text { acute lesions } \\
\text { single and }>3 \text { vertebral } \\
\text { segments }{ }^{\text {b; }} \text { however, } \\
\text { chronic lesions may be } \\
\text { short or replaced by }\end{array}$ & $\begin{array}{l}\text { Usually central; } \\
\text { may be } \\
\text { unilateral or } \\
\text { even peripheral; } \\
\text { may vary over }\end{array}$ & $\begin{array}{l}\text { Hyperintense and } \\
\text { in } 90 \% \text { associated } \\
\text { with extremely } \\
\text { hyperintense } \\
\text { lesions ("bright }\end{array}$ & $\begin{array}{l}\text { Usually } \\
\text { hypointense in } \\
\text { acute lesions }\end{array}$ & $\begin{array}{l}\text { Present in almost } \\
\text { all acute lesions; } \\
\text { variable pattern; } \\
\text { but "ring- } \\
\text { enhancing" in }\end{array}$ \\
\hline
\end{tabular}




\begin{tabular}{|c|c|c|c|c|c|}
\hline & $\begin{array}{l}\text { long segments of } \\
\text { atrophy or myelomalacia } \\
\text { ("pseudosyrinx") }\end{array}$ & $\begin{array}{l}\text { the length of the } \\
\text { lesion }\end{array}$ & spotty lesions") ${ }^{d}$ & & $30 \%$ \\
\hline NMOSD (MOG) ${ }^{40}$ & $\begin{array}{l}\text { Acute myelitis }>3 \\
\text { vertebral segments; may } \\
\text { occur in any part of the } \\
\text { spinal cord, but caudal } \\
\text { spinal cord in } 75 \% \\
\text { versus } 20 \% \text { of MS } \\
\text { patients }\end{array}$ & $\begin{array}{l}\text { Acute myelitis } \\
\text { associated with } \\
\text { single, but } \\
\text { occasionally } \\
\text { multiple, lesions }\end{array}$ & Hyperintense & $\begin{array}{l}\text { Usually } \\
\text { hypointense }\end{array}$ & $\begin{array}{l}\text { Usually present } \\
\text { but somewhat } \\
\text { less frequent than } \\
\text { NMOSD (AQP4) }\end{array}$ \\
\hline Infarction $^{41}$ & $\begin{array}{l}60 \%>3 \text { vertebral } \\
\text { segments; may be } \\
\text { normal when performed } \\
\text { within first hours after } \\
\text { symptom onset }\end{array}$ & $\begin{array}{l}\text { Variable; } 65 \% \\
\text { associated with } \\
\text { anterior gray } \\
\text { matter specific } \\
\text { lesions ("owl } \\
\text { eyes")'; } 30 \% \\
\text { with } \\
\text { homogenous }\end{array}$ & $\begin{array}{l}\text { Hyperintense; } 40 \% \\
\text { linear "pencil-like" } \\
\text { configuration in } \\
\text { anterior spinal cord }\end{array}$ & $\begin{array}{l}\text { Commonly evolve } \\
\text { into T1 hypointense } \\
\text { lesions over } \\
\text { months }\end{array}$ & $\begin{array}{l}90 \% \text { linear } \\
\text { enhancement on } \\
\text { sagittal images } \\
\text { corresponding to } \\
\text { gray matter spinal } \\
\text { cord distribution }\end{array}$ \\
\hline
\end{tabular}




\begin{tabular}{|c|c|c|c|c|c|}
\hline & & $\begin{array}{l}\text { central gray or } \\
\text { entire spinal } \\
\text { cord cross } \\
\text { section }\end{array}$ & & & \\
\hline Viral myelitis $^{42}$ & $\begin{array}{l}\text { Usually }>3 \text { vertebral } \\
\text { segments }\end{array}$ & $\begin{array}{l}\text { Variable; may } \\
\text { be associated } \\
\text { with "owl eye" } \\
\text { appearance } \\
\text { (enterovirus) or } \\
\text { central spinal } \\
\text { cord } \\
\text { (herpesvirus) }\end{array}$ & Hyperintense & Variable & Variable \\
\hline Sarcoidosis $^{43}$ & $\begin{array}{l}>3 \text { vertebral segments } \\
\text { in most patients }\end{array}$ & $\begin{array}{l}\text { Central or entire } \\
\text { cross sectional } \\
\text { area }\end{array}$ & Hyperintense & $\begin{array}{l}\text { Hypointense in } \\
50 \%\end{array}$ & $\begin{array}{l}\text { Posterior subpial } \\
\text { homogeneous } \\
\text { enhancement } \\
\text { over long } \\
\text { segments of } \\
\text { spinal cord; }\end{array}$ \\
\hline
\end{tabular}




\begin{tabular}{|c|c|c|c|c|c|}
\hline & & & & & $\begin{array}{l}\text { central canal } \\
\text { enhancement } \\
\text { common; "trident } \\
\text { sign" on axial } \\
\text { images; ring- } \\
\text { enhancement not } \\
\text { seen }\end{array}$ \\
\hline $\begin{array}{l}\text { Spondylotic } \\
\text { compressive } \\
\text { myelopath }^{44}\end{array}$ & $\begin{array}{l}\text { Variable; may be }>3 \\
\text { vertebral segments }\end{array}$ & Central & Hyperintense & $\begin{array}{l}\text { May have disc-like } \\
\text { pattern } \\
\text { corresponding to } \\
\text { site of } \\
\text { enhancement }\end{array}$ & $\begin{array}{l}\text { Disc-like "flat } \\
\text { pancake" pattern } \\
\text { of enhancement } \\
\text { at point of } \\
\text { maximum spinal } \\
\text { cord impingement } \\
\text { often present }\end{array}$ \\
\hline Paraneoplastic $^{45}$ & $\begin{array}{l}\text { Usually over multiple } \\
\text { vertebral segments }\end{array}$ & $\begin{array}{l}\text { Symmetrical } \\
\text { "tract-specific" } \\
\text { lesion }\end{array}$ & Hyperintense & Isointense & $\begin{array}{l}\text { Variable; } \\
\text { homogeneous } \\
\text { gadolinium } \\
\text { enhancement in }\end{array}$ \\
\hline
\end{tabular}




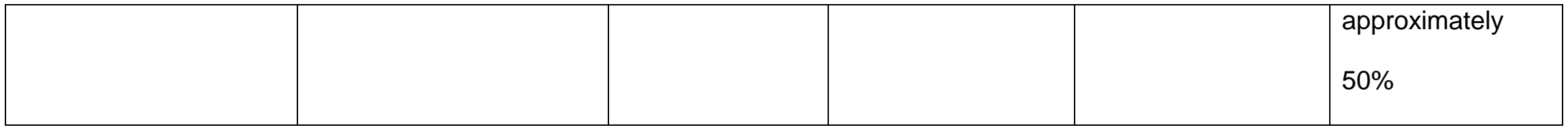

AQP4 = aquaporin-4, MOG = myelin oligodendrocyte glycoprotein, NMOSD = neuromyelitis optica spectrum disorders

${ }^{a}$ Chronic MS lesions may appear confluent on sagittal images leading to radiologic confusion with other conditions associated with longitudinally extensive lesions; axial images usually clarify by revealing multiple central and peripheral conglomerated lesions in MS.

${ }^{b}$ in patients not receiving immunosuppression

${ }^{c}$ reported to occur with equal frequency in acute myelitis associated with NMOSD in one study ${ }^{46}$

dalso may occur in $30 \%$ of spinal cord infarcts 
Figure 1
Click here to download high resolution image
A.a

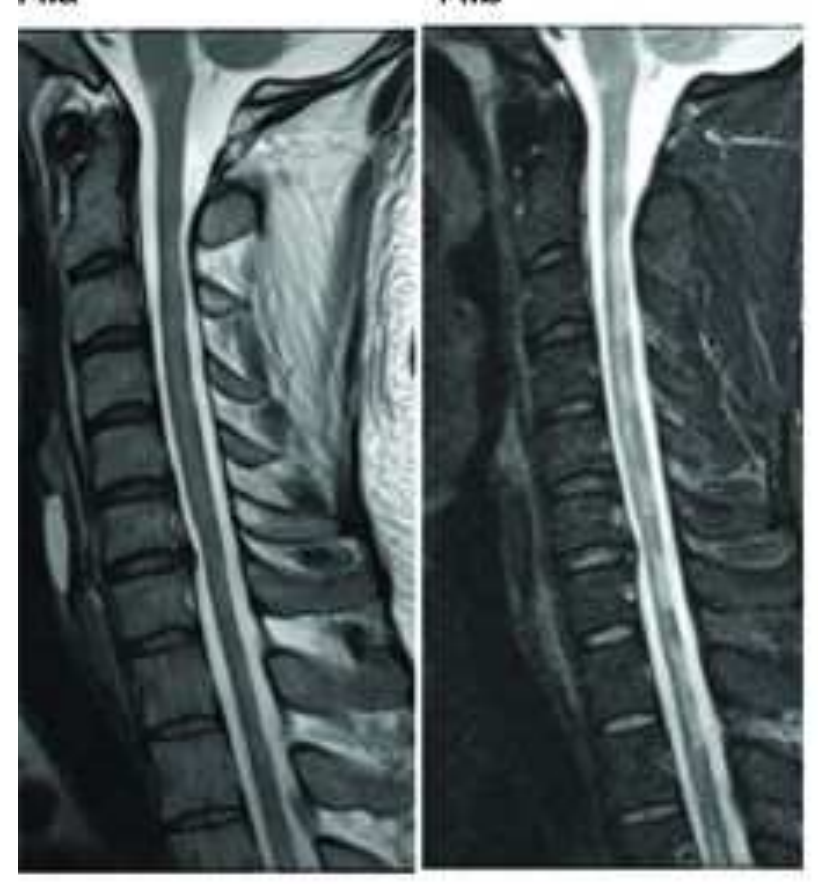

C.a

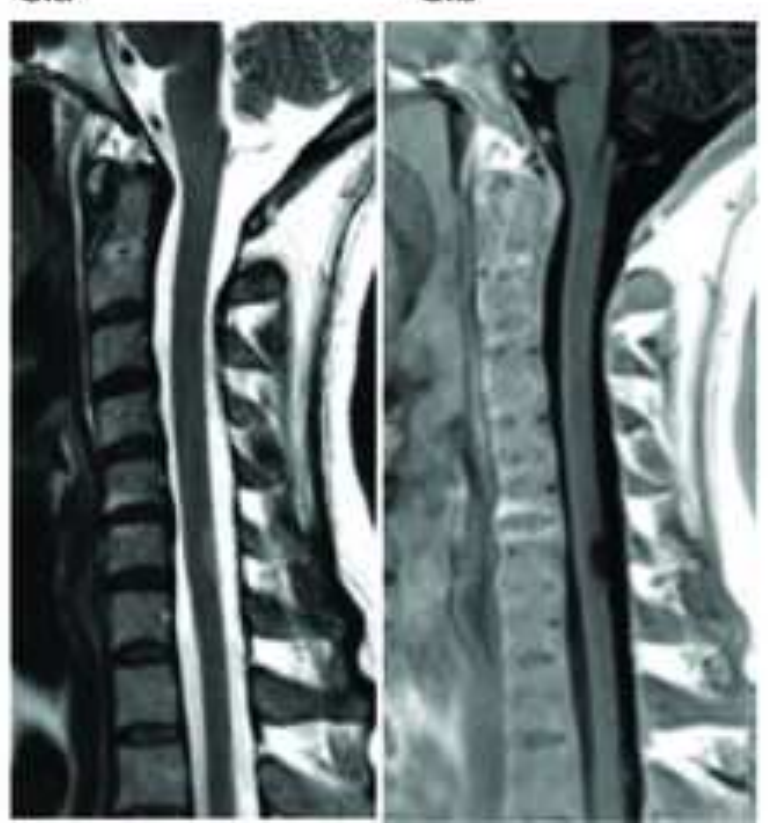

A.b

C.b
B.a

B.b

D.

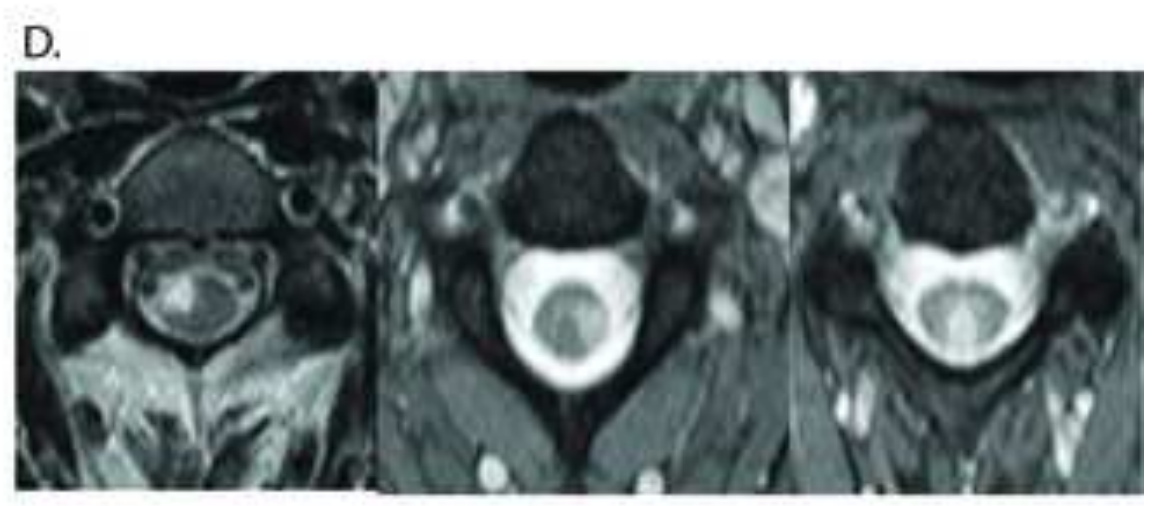

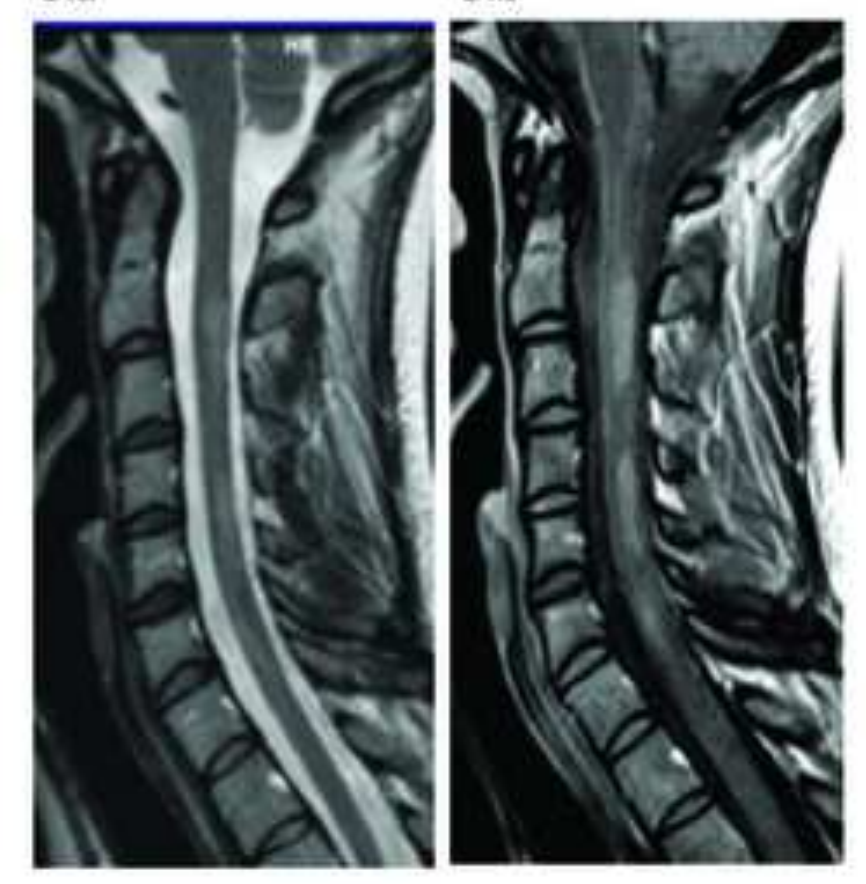

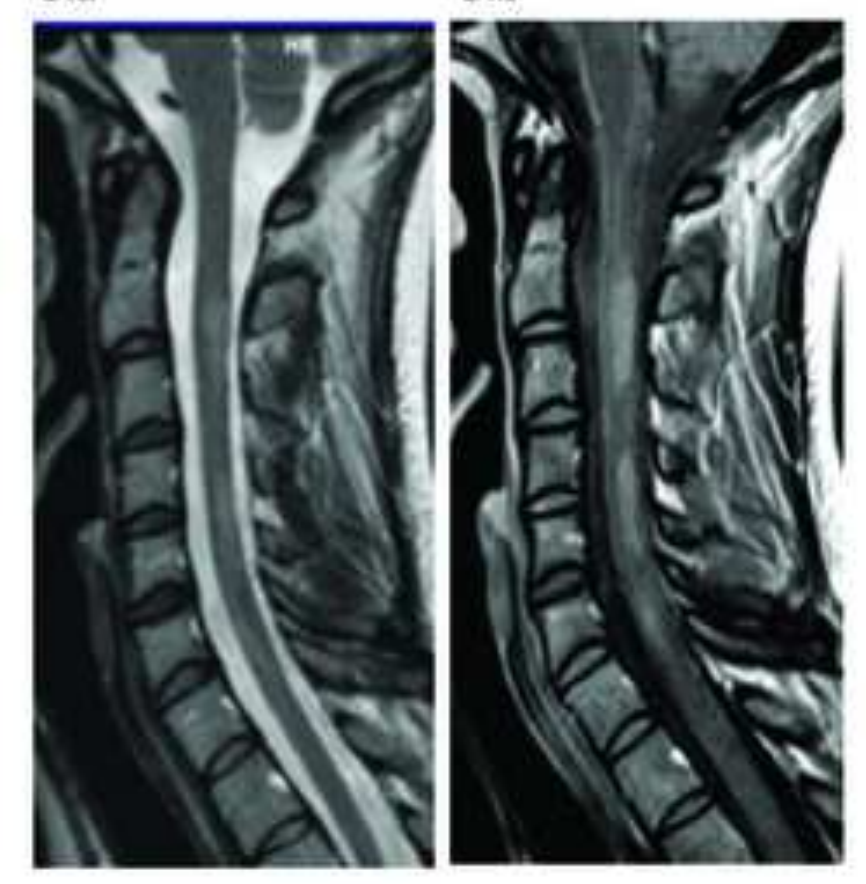



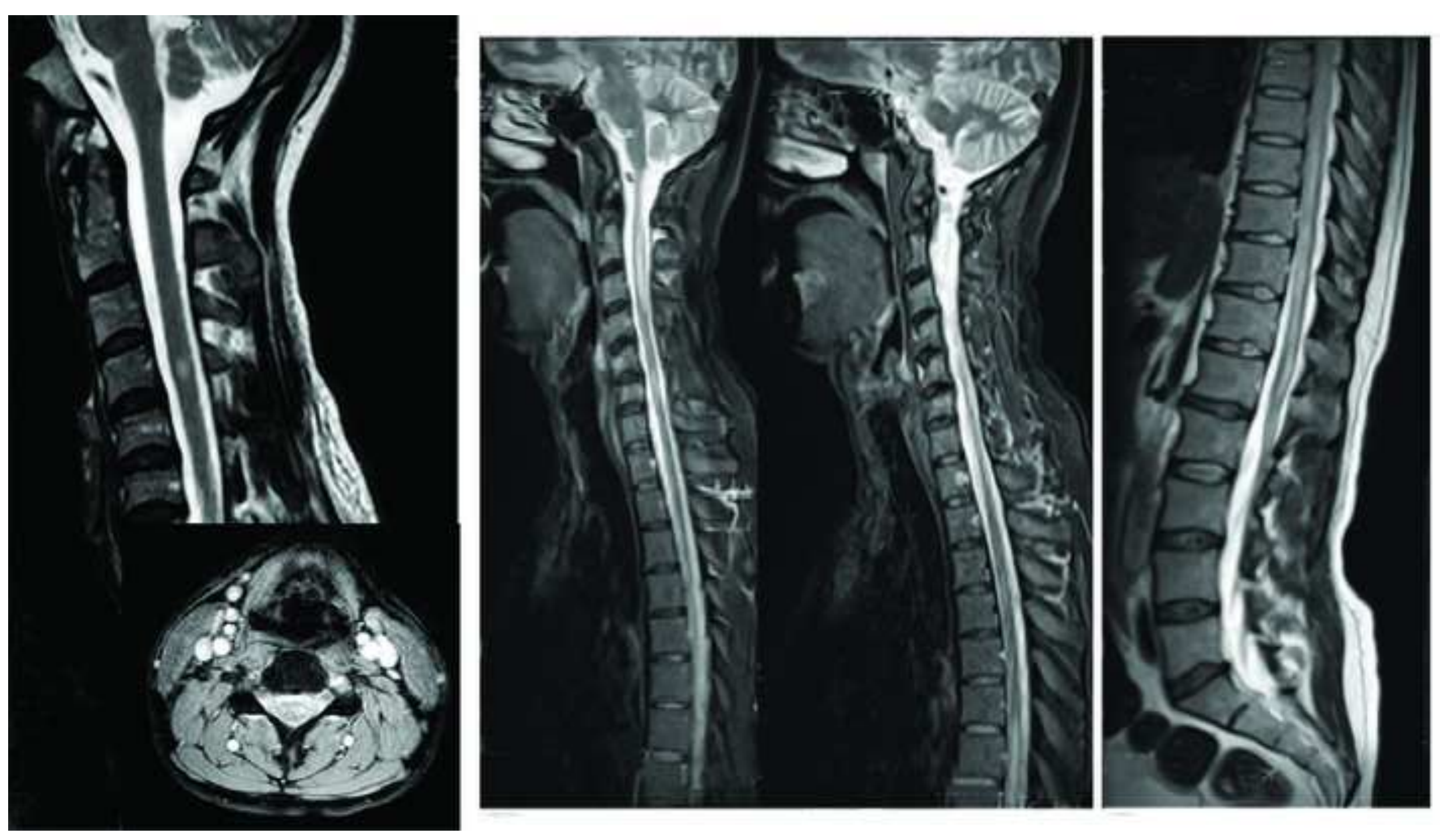

\section{Click here to download high resolution image}



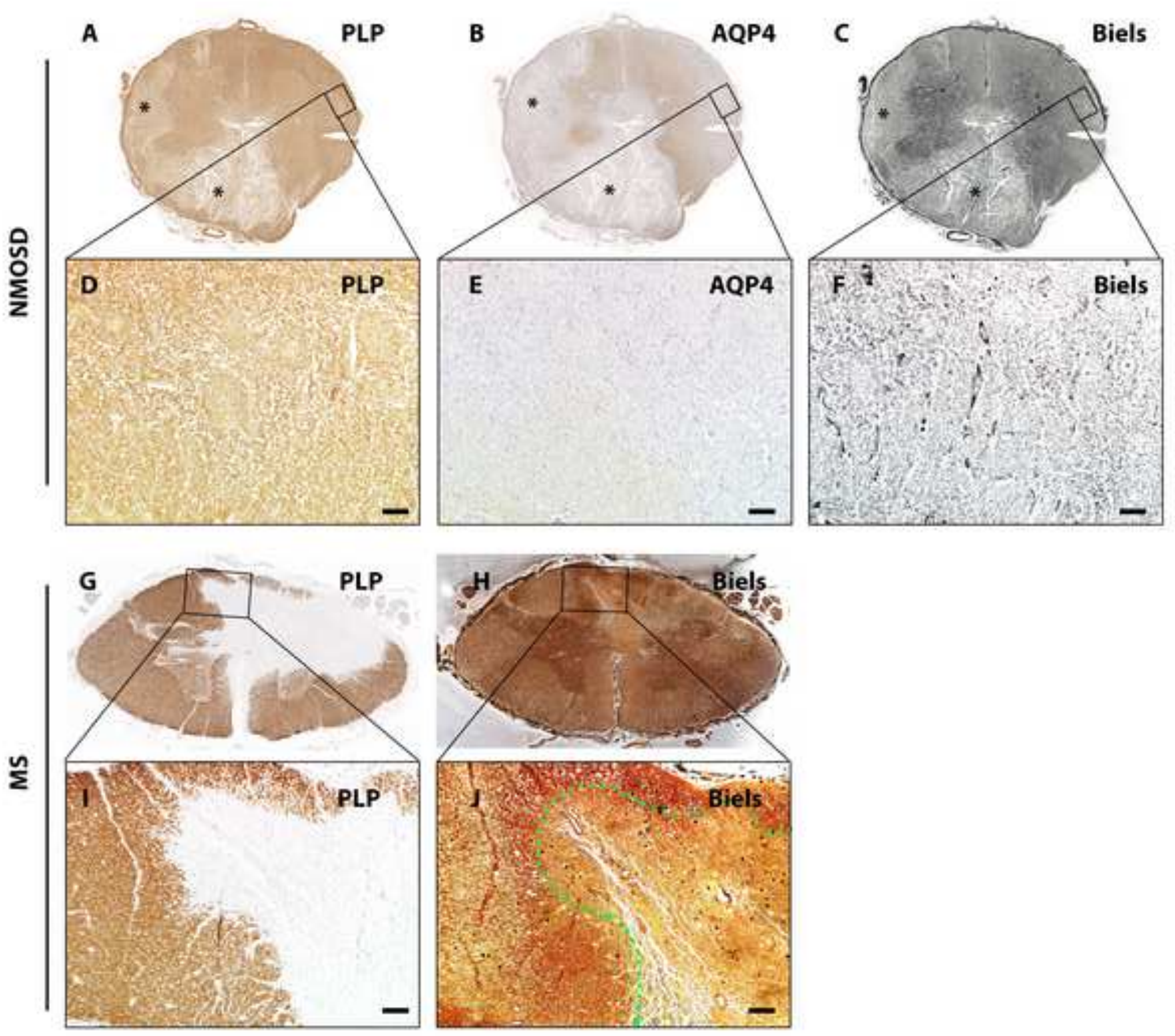